\title{
Adaptively scaling the Metropolis algorithm using expected squared jumped distance*
}

\author{
Cristian Pasarica $^{\dagger} \quad$ Andrew Gelman ${ }^{\ddagger}$
}

January 25, 2005

\begin{abstract}
Using existing theory on efficient jumping rules and on adaptive MCMC, we construct and demonstrate the effectiveness of a workable scheme for improving the efficiency of Metropolis algorithms.

A good choice of the proposal distribution is crucial for the rapid convergence of the Metropolis algorithm. In this paper, given a family of parametric Markovian kernels, we develop an algorithm for optimizing the kernel by maximizing the expected squared jumped distance, an objective function that characterizes the Markov chain under its $d$-dimensional stationary distribution. The algorithm uses the information accumulated by a single path and adapts the choice of the parametric kernel in the direction of the local maximum of the objective function using multiple importance sampling techniques.

We follow a two-stage approach: a series of adaptive optimization steps followed by an MCMC run with fixed kernel. It is not necessary for the adaptation itself to converge. Using several examples, we demonstrate the effectiveness of our method, even for cases in which the Metropolis transition kernel is initialized at very poor values.
\end{abstract}

Keywords: Acceptance rates; Bayesian computation; iterative simulation; Markov chain Monte Carlo; Metropolis algorithm; multiple importance sampling

\section{Introduction}

\subsection{Adaptive MCMC algorithms: motivation and difficulties}

The algorithm of Metropolis et al. (1953) is an important tool in statistical computation, especially in calculation of posterior distributions arising in Bayesian statistics. The Metropolis algorithm evaluates a (typically multivariate) target distribution $\pi(\theta)$ by generating a Markov chain whose stationary distribution is $\pi$. Practical implementations often suffer from slow mixing and therefore inefficient estimation, for at least two reasons: the jumps are too short and therefore simulation moves very slowly to the target distribution; or the jumps end up in low-target areas of the target density, causing the Markov chain to stand still most of the time. In practice, adaptive methods have been proposed in order to tune the choice of the proposal,

\footnotetext{
${ }^{*}$ We thank the National Science Foundation for financial support.

${ }^{\dagger}$ Department of Statistics, Columbia University, New York, NY 10027, pasarica@stat.columbia.edu

${ }^{\ddagger}$ Department of Statistics, Columbia University, New York, NY 10027, gelman@stat.columbia.edu
} 
matching some criteria under the invariant distribution (e.g., Haario, Saksman, and Tamminen, 1999, Laskey and Myers, 2003, Andrieu and Robert, 2001, and Atchadé and Rosenthal, 2003). These criteria are usually defined based on theoretical optimality results, for example for a $d$-dimensional normal target distribution the optimal scaling of the jumping kernel is $c_{d}=2.4 / \sqrt{d}$ (Gelman, Roberts, and Gilks, 1996).

Another approach is to coerce the acceptance probability to a preset value (e.g., 23\%; see Roberts, Gelman, and Gilks, 1997) with covariance kernel set by matching moments; these can be difficult to apply due to the complicated form of target distribution which makes the optimal acceptance probability value or analytic moments difficult to compute. In practice, problems arise for distributions for which the normaltheory optimal scaling results do not apply, and for high-dimensional target distributions where initial optimization algorithms cannot find easily the global maximum of the target distribution, yielding a proposal covariance matrix different from the covariance matrix under the invariant distribution.

This paper presents an algorithm for improving the efficiency of Metropolis algorithms by optimizing the expected squared jumped distance (ESJD), which is the average of the acceptance probability multiplied by the squared distance of the jumping proposal. Optimizing this measure is equivalent to minimizing first-order autocorrelation, an idea that has been proposed by many researchers, but we go further in two ways: first, the ESJD is a more stable quantity than related measures such as autocorrelation or empirical acceptance rates and thus can be optimized more effectively in a small number of iterations; and second, we use a multiple importance sampling estimate that allows us to optimize the ESJD using a series of simulations from different jumping kernels. As a result, adaptation can proceed gradually while making use of information from earlier steps.

Unfortunately, fully adaptive proposal Metropolis algorithms do not in general produce simulations from the target distribution: the Markovian property or time-homogeneity of the transition kernel is lost, and ergodicity can be proved only under some very restrictive conditions (see Haario, Saksman, and Tamminen, 2001, Holden, 1998, and Atchadé and Rosenthal, 2003). Adaptive methods that preserve the Markovian properties using regeneration have the challenge of estimation of regeneration times, which is difficult for algorithms other than independence chain Metropolis (see Gilks, Roberts, and Sahu, 1998).

Our algorithm is semi-adaptive in that it adapts the jumping kernel several times as part of a burn-in phase, followed by an MCMC run with fixed kernel. After defining the procedure in general terms in Section 2, we discuss the theory of convergence of the adaptations in Section 3. Our method can work even if the adaptation does not converge (since we run with a fixed kernel after the adaptation stops) but the theory gives us some insight into the progress of the adaptation. We illustrate the method in Section 4 with several examples, including Gaussian kernels from 1 to 100 dimensions, a normal/ $t$ hierarchical model, and a more complicated nonlinear hierarchical model that arose from applied research in biological measurement.

The innovation of this paper is not in the theory of adaptive algorithms but rather in developing a 
particular implementation that is effective and computationally feasible for a range of problems, including those for which the transition kernel is initialized at very poor values.

\subsection{Our proposed method based on expected squared jumped distance}

In this paper we propose a general framework which allows for the development of new MCMC algorithms that are able to approximately optimize among a set of proposed transition kernels $\left\{J_{\gamma}\right\}_{\gamma \in \Gamma}$, where $\Gamma$ is some finite-dimensional domain, in order to explore the target distribution $\pi$.

Measures of efficiency in low dimensional Markov chains are not unique (see Besag and Green, 1993, Gelman, Roberts, and Gilks, 1996, and Andrieu and Robert, 2001). We shall maximize the expected squared jumped distance (ESJD):

$$
\operatorname{ESJD}(\gamma) \triangleq \mathbf{E}_{J_{\gamma}}\left[\left|\theta_{t+1}-\theta_{t}\right|^{2}\right]=2\left(1-\rho_{1}\right) \cdot \operatorname{var}_{\pi}\left(\theta_{t}\right),
$$

for a one-dimensional target distribution $\pi$, and a similar quantity in multiple dimensions (see Section 2.4). Clearly, $\operatorname{var}_{\pi}\left(\theta_{t}\right)$ is a function of the stationary distribution only, thus choosing a transition rule to maximize ESJD is equivalent to minimizing the first order autocorrelation $\rho_{1}$ of the Markov chain. Our algorithm follows these steps:

1. Start the Metropolis algorithm with some initial kernel; keep track of both the Markov chain $\theta_{t}$ and proposals $\theta_{t}^{*}$.

2. After every $T$ iterations, update the covariance matrix of the jumping kernel using the sample covariance matrix, with a scale factor that is computed by optimizing an importance sampling estimate of the ESJD.

3. After some number of the above steps, stop the adaptive updating and run the MCMC with a fixed kernel, treating the previous iterations up to that point as a burn-in.

Although we focus on the ESJD, we derive our method more generally and it can apply to any objective function that can be calculated from the simulation draws.

Importance sampling techniques for Markov chains, unlike for independent variables, typically require the whole path for computing the importance sampling weights, thus making them computationally expensive. We take advantage of the properties of the Metropolis algorithm to construct importance weights that depend only on the current state, and not of the whole history of the chain. The multiple importance sampling techniques introduced in Geyer and Thomson (1992, reply to discussion) and Geyer (1996) help stabilize the variance of the importance sampling estimate over a broad region, by treating observations from different samples as observations from a mixture density. We study the convergence of our method by using 
the techniques of Geyer (1994). Our method can work even if the adaptation does not converge (since we run with a fixed kernel after the adaptation stops) but the theory gives us some insight into the progress of the adaptation.

This paper describes our approach, in particular, the importance sampling method used to optimize the parameters of the jumping kernel $J_{\gamma}(\cdot, \cdot)$ after a fixed number of steps, and illustrates it with several examples. We also compare our procedure with the Robbins-Monro stochastic optimization algorithm (see, for example, Kushner and Yin, 2003). We describe our algorithm in Section 2 in general and in Section 3 discuss implementation with Gaussian kernels. Section 4 includes several examples, and we conclude with discussion and open problems in Section 5.

\section{The adaptive optimization procedure}

\subsection{Notation}

To define Hastings's (1970) version of the algorithm, suppose that $\pi$ is a target density absolutely continuous with respect to Lebesgue measure and let $\left\{J_{\gamma}(\cdot, \cdot)\right\}_{\gamma \in \Gamma}$ be a family of jumping (proposal) kernels. For fixed $\gamma \in \Gamma$ define

$$
\alpha_{\gamma}(x, y)=\min \left\{\frac{J_{\gamma}(y, x) \pi(y)}{\pi(x) J_{\gamma}(x, y)}, 1\right\} .
$$

If we define the off-diagonal density of the Markov process,

$$
p_{\gamma}(x, y)= \begin{cases}J_{\gamma}(x, y) \alpha_{\gamma}(x, y), & x \neq y \\ 0, & x=y\end{cases}
$$

and set

$$
r_{\gamma}(x)=1-\int p_{\gamma}(x, y) d y,
$$

then the Metropolis transition kernel can be written as

$$
\begin{aligned}
K_{\gamma}(x, d y) & =\left(1 \wedge \frac{\pi(y) J_{\gamma}(y, x)}{\pi(x) J_{\gamma}(x, y)}\right) J_{\gamma}(x, d y) \mathbf{1}_{\{x \neq y\}}+\delta_{x}(y)\left(1-\int\left(1 \wedge \frac{\pi(y) J_{\gamma}(y, x)}{\pi(x) J_{\gamma}(x, y)}\right) J_{\gamma}(x, y) d y\right) \\
& =p_{\gamma}(x, y) d y+r_{\gamma}(x) \delta_{x}(d y) .
\end{aligned}
$$

Throughout this paper we use the notation $\theta_{t}^{*}$ for the proposal generated by the Metropolis-Hastings chain under jumping kernel $J_{\gamma}\left(\cdot, \theta_{t}\right)$ and denote by

$$
\Delta_{t} \triangleq \theta_{t}^{*}-\theta_{t},
$$

the proposed jumping distance. Clearly $\theta_{t+1}=\theta_{t}^{*}$ with probability $\alpha\left(\theta_{t}, \theta_{t}^{*}\right)$, and $\theta_{t+1}=\theta_{t}$, with probability $1-\alpha\left(\theta_{t}, \theta_{t}^{*}\right)$. 


\subsection{Optimization of the jumping kernel after one set of simulations}

Following Andrieu and Robert (2001), we define the objective function which we seek to maximize adaptively as

$$
h(\gamma) \triangleq \mathbf{E}\left[H\left(\gamma, \theta_{t}, \theta_{t}^{*}\right)\right]=\iint_{\mathbf{R}^{d} \times \mathbf{R}^{d}} H(\gamma, x, y) \pi(x) J_{\gamma}(x, y) d x d y, \forall \gamma \in \Gamma .
$$

We start our procedure by choosing an initial jumping kernel $J_{\gamma_{0}}(\cdot, \cdot)$ and running the Metropolis-Hastings algorithm for $T$ steps. We can use the $T$ simulation draws $\theta_{t}$ and the proposals $\theta_{t}^{*}$ to construct the empirical ratio estimator of $h(\gamma)$,

$$
\hat{h}_{T}\left(\gamma \mid \gamma_{0}\right) \triangleq \frac{\sum_{t=1}^{T} H\left(\gamma, \theta_{t}, \theta_{t}^{*}\right) \cdot w_{\gamma \mid \gamma_{0}}\left(\theta_{t}, \theta_{t}^{*}\right)}{\sum_{t=1}^{T} w_{\gamma \mid \gamma_{0}}\left(\theta_{t}, \theta_{t}^{*}\right)}, \forall \gamma \in \Gamma
$$

or the mean estimator

$$
h_{T}\left(\gamma \mid \gamma_{0}\right) \triangleq \frac{1}{T} \sum_{t=1}^{T} H\left(\gamma, \theta_{t}, \theta_{t}^{*}\right) \frac{J_{\gamma}\left(\theta_{t}, \theta_{t}^{*}\right)}{J_{\gamma_{0}}\left(\theta_{t}, \theta_{t}^{*}\right)}, \forall \gamma \in \Gamma .
$$

where

$$
w_{\gamma \mid \gamma_{0}}(x, y) \triangleq \frac{J_{\gamma}(x, y)}{J_{\gamma_{0}}(x, y)},
$$

are the importance sampling weights. On the left side of (3) the subscript $T$ emphasizes that the estimate comes from $T$ simulation draws, and we explicitly condition on $\gamma_{0}$ because the importance sampling weights require $J_{\gamma_{0}}$.

We typically choose as objective function the expected squared jumped distance $H\left(\gamma,\left(\theta, \theta^{*}\right)\right)=\| \theta-$ $\theta^{*} \|_{\Sigma^{-1}}^{2} \alpha_{\gamma}\left(\theta, \theta^{*}\right)=\left(\theta-\theta^{*}\right)^{t} \Sigma^{-1}\left(\theta-\theta^{*}\right) \alpha_{\gamma}\left(\theta, \theta^{*}\right)$, where $\Sigma$ is the covariance matrix of the target distribution $\pi$, because maximizing this distance is equivalent with minimizing the first order autocorrelation in covariance norm. We return to this issue and discuss other choices of objective function in Section 2.4. We optimize the empirical estimator (3) using a numerical optimization algorithm such as Brent's (see, e.g., Press et al., 2002) as we further discuss in Section 2.6. In Section 4 we discuss the computation time needed for the optimization.

\subsection{Iterative optimization of the jumping kernel}

If the starting point is not in the neighborhood of the optimum, then an effective strategy is to iterate the optimization procedure, both to increase the amount of information used in the optimization and to use more effective importance sampling distributions. The iteration allows us to get closer and not rely too strongly on our starting distribution. We explore the effectiveness of the iterative optimization in several examples in Section 4. In our algorithm, the "pilot data" used to estimate $h$ will come from a series of different 
jumping kernels. The function $h$ can be estimated using the method of multiple importance sampling (see Hesterberg, 1995), yielding the following algorithm based on adaptively updating the jumping kernel after steps $T_{1}, T_{1}+T_{2}, T_{1}+T_{2}+T_{3}+\cdots$. For $k=1,2,3, \ldots$,

1. Run the Metropolis algorithm for $T_{k}$ steps according to jumping rule $J_{\gamma_{k}}(\cdot, \cdot)$. Save the sample and proposals, $\left(\theta_{k 1}, \theta_{k 1}^{*}\right), \ldots,\left(\theta_{k T_{k}}, \theta_{k T_{k}}^{*}\right)$.

2. Find the maximum $\gamma_{k+1}$ of the empirical estimator $h_{T_{1}+\cdots+T_{k}}\left(\gamma \mid \gamma_{k}, \ldots, \gamma_{1}\right)$, defined as

$$
\hat{h}_{T_{1}+\cdots+T_{k}}\left(\gamma \mid \gamma_{k}, \ldots, \gamma_{1}\right)=\frac{\sum_{i=1}^{k} \sum_{t=1}^{T_{i}} H\left(\gamma, \theta_{i t}, \theta_{i t}^{*}\right) \cdot w_{\gamma \mid \gamma_{k}, \ldots, \gamma_{1}}\left(\theta_{i t}, \theta_{i t}^{*}\right)}{\sum_{i=1}^{k} \sum_{t=1}^{T_{i}} w_{\gamma \mid \gamma_{k}, \ldots, \gamma_{1}}\left(\theta_{i t}, \theta_{i t}^{*}\right)},
$$

where the multiple importance sampling weights are

$$
w_{\gamma \mid \gamma_{j}, \ldots, \gamma_{1}}\left(\theta, \theta^{*}\right) \triangleq \frac{J_{\gamma}\left(\theta, \theta^{*}\right)}{\sum_{i=1}^{j} T_{i} J_{\gamma_{i}}\left(\theta, \theta^{*}\right)}, j=1, \ldots, k .
$$

We are treating the samples as having come from a mixture of $k$ distributions. The weights satisfy the condition $\sum_{i=1}^{k} \sum_{t=1}^{T_{i}} w_{\gamma \mid \gamma_{k}, \ldots, \gamma_{1}}\left(\theta_{i t}, \theta_{i t}^{*}\right)=1$ and are derived from the individual importance sampling weights by substituting $J_{\gamma}=\omega_{\gamma \mid \gamma_{j}} J_{\gamma_{j}}$ in the numerator of (7). With independent multiple importance sampling, these weights are optimal in the sense that they minimize the variance of the empirical estimator (see Veach and Guibas, 1995, Theorem 2), and our numerical experiments indicate that this greatly improves the convergence of our method. Since step 2 is nested within a larger optimization procedure, if suffices to run only a few steps of an optimization algorithm, there is no need to find the local optimum since it will be altered at next step anyway. Also, it is not always necessary to keep track of the whole chain and proposals, quantities that can become be computationally expensive for high dimensional distributions. For example, in the case of random walk Metropolis and ESJD objective function it is enough to keep track of the jumped distance in covariance norm and the acceptance probability to construct the adaptive empirical estimator. We further discuss these issues in Section 3.

\subsection{Choices of the objective function}

We focus on optimizing the expected squared jumped distance (ESJD), which in one dimension is defined as,

$$
\begin{aligned}
\operatorname{ESJD}(\gamma) & =\mathbf{E}_{J_{\gamma}}\left[\left|\theta_{t+1}-\theta_{t}\right|^{2}\right]=\mathbf{E}_{J_{\gamma}}\left[\mathbf{E}_{J_{\gamma}}\left[\left|\theta_{t+1}-\theta_{t}\right|^{2} \mid\left(\theta_{t}, \theta_{t}^{*}\right)\right]\right] \\
& =\mathbf{E}\left[\left|\theta_{t}^{*}-\theta_{t}\right|^{2} \alpha_{\gamma}\left(\theta_{t}, \theta_{t}^{*}\right)\right]=2\left(1-\rho_{1}\right) \cdot \operatorname{var}_{\pi}\left(\theta_{t}\right)
\end{aligned}
$$

and corresponds to the objective function $H\left(\gamma, \theta, \theta^{*}\right)=\left(\theta-\theta^{*}\right)^{2} \alpha_{\gamma}\left(\theta, \theta^{*}\right)$. Maximizing the ESJD is equivalent to minimizing first order autocorrelation, which is a convenient approximation to maximizing efficiency, as we have discussed in Section 1.2. 
For $d$-dimensional targets, we scale the expected squared jumped distance by the covariance norm and define the ESJD as

$$
\operatorname{ESJD}(\gamma) \triangleq \mathbf{E}_{J_{\gamma}}\left[\left\|\theta_{t+1}-\theta_{t}\right\|_{\Sigma^{-1}}^{2}\right]=\mathbf{E}\left[\left\|\theta_{t}^{*}-\theta_{t}\right\|_{\Sigma^{-1}}^{2} \alpha_{\gamma}\left(\theta_{t}, \theta_{t}^{*}\right)\right]
$$

This corresponds to the objective function, $H\left(\gamma, \theta, \theta^{*}\right)=\left\|\theta-\theta^{*}\right\|_{\Sigma^{-1}}^{2} \alpha_{\gamma}\left(\theta, \theta^{*}\right)=\left(\theta-\theta^{*}\right)^{t} \Sigma^{-1}\left(\theta-\theta^{*}\right) \alpha_{\gamma}\left(\theta, \theta^{*}\right)$, where $\Sigma$ is the covariance matrix of the target distribution $\pi$. The adaptive estimator (6) then becomes,

$$
\hat{h}_{T_{1}+\cdots+T_{k}}\left(\gamma \mid \gamma_{k}, \gamma_{k-1}, \ldots, \gamma_{1}\right) \triangleq \frac{\sum_{i=1}^{k} \sum_{t=1}^{T_{i}}\left\|\Delta_{i t}\right\|_{\Sigma^{-1}}^{2} \alpha_{\gamma_{i}}\left(\theta_{i t}, \theta_{i t}^{*}\right) \cdot w_{\gamma \mid \gamma_{k}, \ldots, \gamma_{1}}\left(\theta_{i t}, \theta_{i t}^{*}\right)}{\sum_{i=1}^{k} \sum_{t=1}^{T_{i}} w_{\gamma \mid \gamma_{k}, \ldots, \gamma_{1}}\left(\theta_{i t}, \theta_{i t}^{*}\right)} .
$$

Maximizing the ESJD in covariance norm is equivalent to minimizing the lag-1 correlation of the $d$-dimensional process in covariance norm,

$$
\operatorname{ESJD}(\gamma)=\mathbf{E}_{J_{\gamma}}\left[\left\|\theta_{t}\right\|_{\Sigma^{-1}}^{2}\right]-\mathbf{E}_{J_{\gamma}}\left[<\theta_{t+1}, \theta_{t}>_{\Sigma^{-1}}\right]
$$

When $\Sigma$ is unknown, we can use a current estimate in defining the objective function at each step. We illustrate in Sections 4.2 and 4.4 .

For other choices of objective function in the MCMC literature, see Andrieu and Robert (2001). In this paper we shall consider two optimization rules: (1) maximizing the ESJD (because of its property of minimizing the first order autocorrelation) and (2) coercing the acceptance probability (because of its simplicity).

\subsection{Convergence properties}

For fixed jumping kernel, under conditions on $\pi$ and $J_{\gamma}$ such that the Markov chain $\left(\theta_{t}, \theta_{t}^{*}\right)$ is irreducible and aperiodic (see Meyn and Tweedie, 1993), the ratio estimator $\hat{h}_{T}$ converges to $h$ with probability 1 . In order to prove convergence of the maximizer of $\hat{h}_{T}$ to the maximizer of $h$, some stronger properties are required.

Proposition 1. Let $\left\{\left(\theta_{t}, \theta_{t}^{*}\right)\right\}_{t=1: T}$ be the Markov chain and set of proposals generated by the MetropolisHastings algorithm under transition kernel $J_{\gamma_{0}}(\cdot, \cdot)$. If the chain $\left\{\left(\theta_{t}, \theta_{t}^{*}\right)\right\}$ is irreducible, and $\hat{h}_{T}\left(\cdot \mid \gamma_{0}\right)$ and $h$ are concave and twice differentiable everywhere, then $\hat{h}_{T}\left(\cdot \mid \gamma_{0}\right)$ converges to $h$ uniformly on compacts with probability 1 and the maximizers of $\hat{h}_{T}\left(\cdot \mid \gamma_{0}\right)$ converge to the unique maximizer of $h$.

Proof. The proof is a consequence of well-known theorems of convex analysis stating that convergence on a dense set implies uniform convergence and consequently convergence of the maximizers, and can be found in Geyer and Thompson (1992).

In general, it is difficult to check the concavity assumption for the empirical ratio estimator, but we can prove convergence for the mean estimator.

Proposition 2. Let $\left\{\left(\theta_{t}, \theta_{t}^{*}\right)\right\}_{t=1: T}$ be the Markov chain and set of proposals generated by the MetropolisHastings algorithm under transition kernel $J_{\gamma_{0}}(\cdot, \cdot)$. If the chain $\left\{\left(\theta_{t}, \theta_{t}^{*}\right)\right\}$ is irreducible, and the mapping

$$
\gamma \rightarrow H(\gamma, x, y) J_{\gamma}(x, y), \forall \gamma \in \Gamma
$$


is continuous, and for every $\gamma \in \Gamma$ there is a neighborhood $B$ of $\gamma$ such that

$$
\mathbf{E}_{J_{\gamma_{0}}}\left[\sup _{\phi \in B} H\left(\phi, \theta_{t}, \theta_{t}^{*}\right) \frac{J_{\phi}\left(\theta_{t}, \theta_{t}^{*}\right)}{J_{\gamma_{0}}\left(\theta_{t}, \theta_{t}^{*}\right)}\right]<\infty,
$$

then $h_{T}\left(\cdot \mid \gamma_{0}\right)$ converges to $h$ uniformly on compact sets with probability 1.

Proof. See Appendix.

The convergence of the maximizer of $h_{T}$ to the maximizer of $h$ is attained under the additional conditions of Geyer (1994).

Theorem. (Geyer, 1994, Theorem 4) Assume that $\left(\gamma_{T}\right)_{T}, \gamma_{*}$ are the unique maximizers of $\left(h_{T}\right)_{T}$ and $h$, respectively and they are contained in a compact set. If there exist a sequence $\epsilon_{T} \rightarrow 0$ such that $h_{T}\left(\gamma_{T} \mid \gamma_{0}\right) \geq \sup _{T}\left(h_{T}\left(\gamma_{T} \mid \gamma_{0}\right)\right)-\epsilon_{T}$, then $\gamma_{T} \rightarrow \gamma_{*}$.

Proposition 3. If the chain $\left\{\left(\theta_{t}, \theta_{t}^{*}\right)\right\}$ is irreducible and the objective function is the expected squared jumped distance, $H(\gamma, x, y)=\|y-x\|_{\Sigma^{-1}}^{2} \alpha_{\gamma}(x, y)$, then the mean empirical estimator $h_{T}\left(\gamma \mid \gamma_{0}\right)$ converges uniformly on compact sets for the case of random walk Metropolis algorithm with jumping kernel $J_{\gamma, \Sigma}\left(\theta_{*}, \theta\right) \approx$ $\exp \left(-\frac{1}{2 \gamma^{2}}\left\|\theta-\theta^{*}\right\|_{\Sigma^{-1}}^{2}\right)$.

Proof. See Appendix.

Remark. We used both the mean and the ratio estimator for our numerical experiments, but the convergence appeared to be faster and the estimates more stable for the ratio estimator (see Remark 1 below for more details).

\subsection{Practical optimization issues}

Remark 1. The motivation for the ratio estimator (3) is that it preserves the range of the objective function, for example constraining the acceptance probability to the range $[0,1]$, and has a lower variance than the mean estimator if the correlation between the numerator and denominator is sufficiently high (see Cochran, 1977). Other choices for the empirical estimator include the mean estimator $h_{T}$ and estimators that use control variates that sum to 1 to correct for bias (see, for example, the regression and difference estimators of Hesterberg, 1995).

Multiple importance sampling is intended to give high weights to individual jumping kernels that are near the optimum. For more choices for the multiple importance sampling weights, see Veach and Guibas (1995).

Remark 2. For the usual symmetric kernels (e.g., normal, $t$, Cauchy) and objective functions it is straightforward to derive analytical first and second order derivatives and run a few steps of a maximization algorithm which incorporates the knowledge of the first and second derivative (see, e.g., Press et al., 2002) for $\mathrm{C}$ code or the function optim() in R). If analytic derivatives do not exist or are expensive to compute, then one can perform a grid maximization centered on the current estimated optimum. 
Remark 3. Guidelines that ensure fast convergence of the importance sampling estimator $I_{n}(h)=$ $\sum_{i=1}^{n} h\left(X_{i}\right) \frac{g_{\gamma}\left(X_{i}\right)}{g_{\gamma_{0}}\left(X_{i}\right)}$ of $I(h)=\mathbf{E}_{g_{\gamma}}[h(X)]$ based on the proposal distribution $g_{\gamma_{0}}(\cdot)$ are presented in Robert and Casella (1998): the importance sampling distribution $g_{\gamma_{0}}$ should have heavier tails then the true distribution; minimizing the variance of importance weights minimizes the variance of $I_{n}(h)$.

\section{Implementation with Gaussian kernel}

For the case of a random walk Metropolis algorithm with Gaussian proposal density $J_{\gamma, \Sigma}\left(\theta_{*}, \theta\right) \approx \exp \left(-\frac{1}{2 \gamma^{2}}\left\|\theta-\theta^{*}\right\|_{\Sigma^{-1}}^{2}\right)$, the adaptive empirical estimator (8) of the ESJD is

$$
\hat{h}_{T_{1}+\cdots+T_{k}}\left(\gamma \mid \gamma_{k}, \gamma_{k-1}, \ldots, \gamma_{1}\right) \triangleq \frac{\sum_{i=1}^{k} \sum_{t=1}^{T_{i}}\left\|\Delta_{i t}\right\|_{\Sigma_{i}^{-1}}^{2} \alpha\left(\theta_{i t}, \theta_{i t}^{*}\right) \cdot w_{\gamma \mid \gamma_{k}, \ldots, \gamma_{1}}\left(\left\|\Delta_{i t}\right\|_{\Sigma_{i}-1}^{2}\right)}{\sum_{i=1}^{k} \sum_{t=1}^{T_{i}} w_{\gamma \mid \gamma_{k}, \ldots, \gamma_{1}}\left(\left\|\Delta_{i t}\right\|_{\Sigma_{i}^{-1}}^{2}\right)}
$$

where

$$
w_{\gamma \mid \gamma_{k}, \ldots, \gamma_{1}}(x)=\frac{\frac{1}{\gamma^{d}} \exp \left(-\frac{x}{2 \gamma^{2}}\right)}{\sum_{i=1}^{k} T_{i} \frac{1}{\gamma_{i}^{d}} \exp \left(-\frac{x}{2 \gamma_{i}^{2}}\right)} .
$$

For computational purposes, we program the Metropolis algorithm so that it gives as output the proposed jumping distance in covariance norm $\left\|\Delta_{i t}\right\|_{\Sigma_{i}^{-1}}$ and the acceptance probability. This reduces the memory allocation for the optimization problem to one dimension, and the reduction is extremely important high dimensions where the alternative is to store $d \times T$ arrays. We give here a version of our optimization algorithm that keeps track only of the jumped distance in covariance norm, the acceptance probability, and the sample covariance matrix.

1. Choose a starting covariance matrix $\Sigma_{0}$ for the Metropolis algorithm, for example a numerical estimation of the covariance matrix of the target distribution.

2. Choose starting points for the simulation and some initial scaling for the jumping kernel, for example $c_{d}=2.4 / \sqrt{d}$. Run the algorithm for $T_{1}$ iterations, saving the simulation draws $\theta_{1 t}$, the proposed jumping distances $\left\|\Delta_{1 t}\right\|_{\Sigma_{0}^{-1}}$ in covariance norm, and the acceptance probabilities $\alpha\left(\theta_{1 t}, \theta_{1 t}^{*}\right)$. Optionally, construct a vector consisting of the denominator of the multiple importance sampling weights and discard the sample $\theta_{1 t}$.

3. For $k>1$, run the Metropolis algorithm using jumping kernel $J_{\gamma_{k} \Sigma_{k}}$. Update the covariance matrix using the iterative procedure

$\Sigma_{k+1}(i, j)=\left(1-\frac{T_{k}}{T_{\text {total }}}\right) \Sigma_{k}(i, j)+\frac{1}{T_{\text {total }}}\left(\left(T_{\text {total }}-T_{k}\right) \bar{\theta}_{k-1, i} \bar{\theta}_{k-1, j}-T_{\text {total }} \bar{\theta}_{k i} \bar{\theta}_{k j}+\sum_{t=1}^{T_{k}} \theta_{k t} \theta_{j t}\right), i, j=1, \ldots, d$

where $T_{\text {total }}=T_{1}+\cdots+T_{k}$, and update the scaling using the adaptive algorithm. We also must keep track of the $d$-dimensional mean, but this is not difficult since it satisfies a simple recursion equation. Optionally, iteratively update the denominator of the multiple sampling weights. 
4. Discard the sample $\theta_{k t}$ and repeat the above step.

The updated covariance $\Sigma_{k+1}$ might not be positive definite. In this situation we recommend using a eigenvalue decomposition of the updated covariance, setting the minimum eigenvalue to a small positive value, and rounding up the smaller eigenvalues to this minimum value.

In updating the covariance matrix we can also use the greedy-start procedure using only the accepted jumps (see Haario et al., 1999). For random walk Metropolis, analytic first and second order derivatives are helpful in the implementation of the optimization step (2) (e.g., using a optimization method), and can be derived analytically. If we update the scaling jumping kernel at each step of the iteration using Newton's method,

$$
\gamma_{k+1}=\gamma_{k}-\frac{\hat{h}_{k}^{\prime}\left(\gamma_{k} \mid \gamma_{k}, \ldots, \gamma_{1}\right)}{\hat{h}_{k}^{\prime \prime}\left(\gamma_{k} \mid \gamma_{k}, \ldots, \gamma_{1}\right)},
$$

the scaling parameter $\gamma$ will converge fast in a neighborhood of the true maximum; otherwise bounds on parameters are required in order to implement it successfully. In our examples, we have had success updating the jumping kernel every 50 iterations of the Metropolis algorithm, until approximate convergence. At this point the MCMC algorithm is ready for its "production" run.

\section{Examples}

In our first three examples we use target distributions and proposals for which optimal jumping kernels have been proposed in the MCMC literature to demonstrate that our optimization procedure is reliable. We then apply our method on two applications of Bayesian inference using Metropolis and Gibbs-Metropolis updating.

\subsection{Independent normal target distribution, $d=1, \ldots, 100$}

We begin with the multivariate normal target distribution in $d$ dimensions with identity covariance matrix, for which the results from Gelman, Roberts and Gilks (1996) and Roberts, Gelman and Gilks (1997) apply regarding the choice of optimal scaling. This example provides some guidelines regarding the speed of convergence, the optimal sample size, and the effectiveness of our procedure for different dimensions. In our experiments, our approach outperforms the stochastic Robbins-Monro algorithm, as implemented by Atchadé and Rosenthal (2003).

Figure 1 shows the convergence of the adaptive optimization procedure for dimensions $d=1,10,25,50$, and 100 as well as the corresponding values of the multiple importance sampling estimator of ESJD and average acceptance probability.

Insert Figure 1 here "Optimizing ESJD" 
When starting from very small values, the estimated optimal scale shows some initial high upward jumps, because the importance sampling ratio can be unbounded Convergence to the optimal scaling is achieved in 20 steps with sample size $T=50 \times 20=1000$ for dimension $d$ less then 50 . For dimension $d=100$, reliable convergence requires 30 or more steps of 50 iterations each.

In order to compare our algorithm with the stochastic Robbins-Monro algorithm, we also coerced the acceptance probability by estimating the average acceptance probability using the objective function $H(x, y)=\alpha_{\gamma}(x, y)$ and then minimizing a quadratic loss function $h(\gamma)=\left(\iint \alpha_{\gamma}(x, y) J_{\gamma}(x, y) d x d y-\alpha_{*}\right)^{2}$, where $\alpha_{*}$ is defined as the acceptance rate corresponding to the Gaussian kernel that minimizes the first-order autocorrelation.

Insert Figure 2 here "Coerced probability method"

The convergence of the algorithm coercing the acceptance probability is faster then when maximizing ESJD, which we attribute to the fact that the acceptance probability is less variable then ESJD, thus easier to estimate.

A comparison of our method with the stochastic Robbins-Monro algorithm implemented by Atchadé and Rosenthal (2003, Graph 2), shows that our method converges faster and does not encounter the problems of the stochastic algorithm, which always goes in the first steps to a very low value and then converges from below to the optimal value. It is generally better to overestimate than to underestimate the optimal scaling. Even when jumps are not accepted, our importance sampling estimate uses the information in the attempted jumps via the acceptance probabilities.

To show that our method converges also in extreme cases, we apply our method with two starting values of $(0.01,50) \times 2.4 / \sqrt{d}$ for $d=25$. We use an optimization procedure that is a combination of golden search and successive parabolic interpolation (see Brent, 1973) on the interval $[0.01,100]$.

Insert Figure 3 here "Extreme starting points"

\subsection{Correlated normal target distribution}

We next illustrate adaptive scaling for a target distribution with unknown covariance matrix. We consider a two-dimensional target distribution with covariance $\Sigma=\left(\begin{array}{cc}100 & 9 \\ 9 & 1\end{array}\right)$. A choice for the covariance matrix of the initial Gaussian proposal is the inverse of the Hessian, $-\nabla^{2} \log (\pi)$, computed at the maximum of $\pi$. Unfortunately, numerical optimization methods can perform very badly for high dimensions when the starting point of the algorithm is not close to the maximum. Even for such a simple distribution, starting the BFGS optimization algorithm far from the true mode might not find the global maximum, resulting in a bad initial proposal covariance matrix $\Sigma_{0}$. To represent this possibility, we start here with an independent 
proposal $\Sigma_{0}=\left(\begin{array}{cc}25 & 0 \\ 0 & 1\end{array}\right)$. Figure 4 shows the performance of our algorithm; approximate convergence is achieved in 20 steps.

Insert Figure 4 here "Convergence vs. step number"

\subsection{Mixture target distribution}

We consider now a target distribution that is a mixture of Gaussians with parameters $\mu_{1}=-5.0, \sigma_{1}^{2}=1.0$, $\mu_{2}=5.0, \sigma_{2}^{2}=2.0$ and weights $(\lambda=0.2,1-\lambda)$. The purpose of this example is twofold: first to illustrate that for bimodal distribution the optimal scaling is different from the Gaussian results $c_{d}=2.4 / \sqrt{d}$, and second to compare our method with the stochastic Robbins-Monro algorithm of Andrieu and Robert (2001, Section 7.1) where the acceptance probability was coerced to $40 \%$.

We compare the results of our method given two objective functions, coercing the acceptance probability to $44 \%$ and maximizing the ESJD, in terms of convergence and efficiency. We also compare the speed of the stochastic Robbins-Monro algorithm with the convergence speed of our adaptive optimization procedure.

Insert Figure 5 here "ESJD vs coerced acceptance probability method"

The convergence to the "optimal" acceptance probability for the coerced probability method is attained in 1000 iterations for all starting values, an improvement over the approximately 10000 iterations required under the stochastic optimization algorithm (see Andrieu and Robert, 2001, Figure 6). Maximizing ESJD yields an optimal scaling of $\gamma=9.0$, and a comparison of the correlation structure $\rho_{t}$ (the bottom two graphs of Figure 5) at the optimal scales determined by the two objective functions shows that the autocorrelation decreases much faster for the optimal scale which maximizes ESJD, thus making the ESJD a more appropriate efficiency measure.

\subsection{6-dimensional nonlinear model}

We next consider an applied example - a model for serial dilution assays from Gelman, Chew, and Shnaidman (2004),

$$
\begin{aligned}
y_{i} & \sim N\left(g\left(x_{i}, \beta\right),\left(\frac{g\left(x_{i}, \beta\right)}{A}\right)^{2 \alpha} \sigma_{y}^{2}\right) \\
x_{i} & =d_{i} \cdot x_{j}^{\text {init }}(i),
\end{aligned}
$$

where $g(x, \beta)=\beta_{1}+\beta_{2} /\left(1+\left(x / \beta_{3}\right)^{-\beta_{4}}\right)$. For each sample $j$, we model

$\log x_{j}^{i n i t} \sim N\left(\log \left(d_{j}^{i n i t} \cdot \theta_{j}\right),\left(\sigma^{i n i t}\right)^{2}\right)$, for the standard sample $j=0$

$x_{j}^{i n i t}=\theta_{j}$, for the unknown samples $j=1, \ldots, 10$. 
The constant $A$ is arbitrary and is set to some value in the middle of the range of the data. The parameter $\sigma^{\text {init }}$ is assumed known, and a vague prior distribution is applied to $\sigma_{y}$ and $\beta$. We estimate the unknown concentrations using data from a single plate with 16 calibration measurements and 8 measurements per unknown sample. We know the initial concentration of standard sample $\theta_{0}$ and the dilutions $d_{i}$, and we need to estimate the 10 unknown concentrations $\theta_{j}$ and the parameters $\beta_{1}, \beta_{2}, \beta_{3}, \beta_{4}, \sigma_{\theta}, \sigma_{y}, \alpha$. For faster convergence the $\theta_{i}$ 's are reparameterized as $\log \eta_{i}=\log \theta_{j}-\log \beta_{3}$. We use BFGS to find the maximum likelihood and start the Metropolis with a Gaussian proposal with the covariance set to the inverse of the Hessian of the log likelihood computed in the maximum. We keep the covariance matrix fixed and optimize only the choice of scaling. After the algorithm converges, we verify that the sample covariance matches the choice of our initial covariance. Despite the complex structure of the target distribution, the adaptive method converges to the theoretical optimal value $c_{d} \approx 2.4 / \sqrt{16}=0.6$ in 30 steps with 50 iterations per step.

\section{Insert Figure 6 here "16-dimensional nonlinear model"}

The computation time is 0.01 seconds per iteration in the Metropolis step, and the optimization step takes an average of 0.04 seconds per step. We update after every 50 iterations and so the optimization adds $0.04 /(50 * 0.01)=8 \%$ to the computing time.

\subsection{Metropolis sampling within Gibbs}

Finally, we apply our method to Metropolis-within-Gibbs sampling with a hierarchical $t$ model applied to the educational testing example from Gelman et al. (2003, Appendix C). The model has the form,

$$
\begin{aligned}
& y_{j} \sim \mathrm{N}\left(\theta_{j}, \sigma_{j}^{2}\right), \sigma_{j} \text { known, for } \mathrm{j}=1, \ldots, 8 \\
& \theta_{j} \mid \nu, \mu, \tau \sim t_{\nu}\left(\mu, \tau^{2}\right), \text { for } j=1, \ldots, 8
\end{aligned}
$$

We use an improper joint uniform prior density for $(\mu, \tau, 1 / \nu)$. To treat $\nu$ as an unknown parameter, the Gibbs sampling simulation includes a Metropolis step for updating $1 / \nu$. Maximizing ESJD, the adaptive procedure converges to the optimal scale $\gamma=0.5$ in 10 steps of 50 iterations each, the same optimal value for the coercing the acceptance probability to $44 \%$.

Insert Figure 7 here "Gibbs within Metropolis"

\section{Discussion}

The proposed adaptive method is straightforward to implement, and maximizing ESJD greatly improves the performance of the Metropolis algorithm in the diverse examples that we have tried. Our algorithm follows similar steps as recent work in adaptive updating of the Metropolis kernel (Haario et al., 1999, 
Andrieu and Robert, 2001, and Atchadé and Rosenthal, 2003), but appears to converge faster, presumably because of the numerical stability of the multiple importance sampling estimate in the context of a Gaussian parametric family of jumping kernels. Coercing the acceptance probability has slightly faster convergence then maximizing the ESJD but not necessarily to an optimal value as we have seen in Figure 5.

The proof of ergodicity of the adaptive chain which adapts both scaling and covariance remains an open theoretical question as does the relationship between ESJD, the eigenvalue structure of the Metropolis kernel, and convergence speed. For Gaussian and independent distributions in high dimensions, samples of the Metropolis algorithm approach an Ornstein-Uhlenbeck process and all reasonable optimization criteria are equivalent (Roberts, Gelman, and Gilks, 1997), but this is not necessarily the case for finite-dimensional problems or adaptive algorithms.

Other issues that arise in setting up the algorithm are the choice of multiple sampling weights, the choice of number of iterations per step, and when to stop the adaptation. In high-dimensional problems, we have optimized the scale of the jumping kernel while updating the covariance matrix using empirical weighting of posterior simulations (as in Haario et al., 1999). We also anticipate that these methods can be generalized to optimize over more general MCMC algorithms, for example slice sampling (Neal, 2003) and Langevin algorithms that involve a translation parameter as well as a scale for the jumping kernel and can achieve higher efficiencies then symmetric Metropolis algorithms (see Roberts and Rosenthal, 2001).

\section{References}

Andrieu, C., and Robert, C. P. (2001). Controlled MCMC for optimal sampling. Technical report, Université Paris-Dauphine.

Atchadé, Y. F., and Rosenthal, J. S. (2003). On adaptive Markov chain Monte Carlo algorithms. Technical report, University of Montreal.

Besag, J., and Green, P. J. (1993). Spatial statistics and Bayesian computation. Journal of the Royal Statistical Society B 55, 25-37.

Brent, R. (1973). Algorithms for Minimization Without Derivatives. Englewood Cliffs, N.J.: Prentice-Hall.

Cochran, W. G. (1977). Sampling Techniques, third edition. New York: Wiley.

Gelfand, A. E., and Sahu, S. K. (1994). On Markov chain Monte Carlo acceleration. Journal of Computational and Graphical Statistics 3, 261-276.

Gelman, A., Roberts, G. O., and Gilks, W. R. (1996). Efficient Metropolis jumping rules. Bayesian Statistics 5, 599-608.

Gelman, A., Chew, G. L., and Shnaidman, M. (2004). Bayesian analysis of serial dilution assays. Biometrics. 
Gelman, A., Carlin, J. B., Stern, H. S., and Rubin, D. B. (2003). Bayesian Data Analysis, second edition. London: Chapman and Hall.

Geweke, J. (1989). Bayesian inference in econometric models using Monte Carlo integration. Econometrica 57, 1317-1339.

Geyer, C. J. (1994). On the convergence of Monte Carlo maximum likelihood calculations. Journal of the Royal Statistical Society B 56, 261-274.

Geyer, C. J. (1996). Estimation and optimization of functions. In Markov Chain Monte Carlo in Practice, eds. W. R. Gilks, S. Richardson, and D. J. Spiegelhalter, 241-258. London: Chapman and Hall.

Geyer, C. J., and Thompson, E. A. (1992). Constrained maximum Monte Carlo maximum likelihood for dependent data (with discussion). Journal of the Royal Statistical Society B 54, 657-699.

Haario, H., Saksman, E., and Tamminen, J. (1999). Adaptive proposal distribution for random walk Metropolis algorithm. Computational Statistics 14, 375-395.

Haario, H., Saksman, E., and Tamminen, J. (2001). An adaptive Metropolis algorithm. Bernoulli 7, $223-242$.

Hastings, W. K. (1970). Monte Carlo sampling methods using Markov chains and their applications. Biometrika 57, 97-109.

Hesterberg, T. (1995). Weighted average importance sampling and defensive mixture distribution. Technometrics 37, 185-194.

Holden, L. (2000). Adaptive chains. Technical report, Norwegian Computing Centre.

Kushner, H. J., and Yin, G. G. (2003). Stochastic Approximation Algorithms and Applications, second edition. New York: Springer-Verlag.

Laskey, K. B., and Myers, J. (2003). Population Markov chain Monte Carlo. Machine Learning.

Metropolis, N., Rosenbluth, A. W., Rosenbluth, M. N., Teller, A. H., and Teller, E. (1953). Equations of state calculations for fast computing machines. Journal of Chemical Physics 21, 1087-1092.

Meyn, S. P., and Tweedie, R. (1993). Markov Chains and Stochastic Stability. New York: Springer-Verlag.

Mira, A. (2001). Ordering and improving the performance of Monte Carlo Markov chains. Statistical Science 16, 340-350.

Neal, R. M. (2003). Slice sampling (with discussion). Annals of Statistics 31, 705-767.

Press, A., Teukolski, S., Vetterling, W., and Flannery, B. (2002). Numerical Recipes. Cambridge University Press.

Robert, C. P., and Casella, G. (1998). Monte Carlo Statistical Methods. New York: Springer.

Roberts, G. O., Gelman, A., and Gilks, W. R. (1997). Weak convergence and optimal scaling of the random walk Metropolis algorithms. Annals of Applied Probability 7, 110-220. 
Roberts, G. O., and Rosenthal, J. S. (2001). Optimal scaling for various Metropolis-Hastings algorithms. Statistical Science 16, 351-367.

R Project (2000). The R project for statistical computing. www.r-project.org.

Tierney, L., and Mira, A. (1999). Some adaptive Monte Carlo methods for Bayesian inference. Statistics in Medicine 18, 2507-2515.

Veach, E., and Guibas, L. (1995). Optimally combining importance sampling techniques for Monte Carlo rendering. SIGRAPH'95 Conference Proceedings, 419-428. 


\section{Appendix}

\section{Proof of Proposition 2}

The chain $\left\{\left(\theta_{t}, \theta_{t}^{*}\right)\right\}$ is a positive Markov chain with invariant probability $\pi(d x) J_{\gamma}(x, d y)$. Given that $\theta_{t}$ is irreducible, it satisfies the conditions of Robert and Casella (1998, Theorem 6.2.5 i), and consequently,

$$
h_{T}\left(\gamma \mid \gamma_{0}\right)=\frac{1}{T} \sum_{t=1}^{T} H\left(\gamma, \theta_{t}, \theta_{t}^{*}\right) w_{\gamma \mid \gamma_{0}}\left(\theta_{t}, \theta_{t}^{*}\right) \rightarrow \iint H(\gamma, x, y) \pi(x) J_{\gamma}(x, y) d x d y, \text { a.s. }, \forall \gamma \in \Gamma .
$$

The next part of the proof is a particular version of Geyer (1994, Theorems 1 and 2), and we reproduce it here for completeness. Taking into account that the union of null sets is a null set, we have that (13) holds a.s. for all $\gamma$ in a countable dense set in $\Gamma$. By the weak convergence of measures,

$$
\inf _{\phi \in B} \frac{1}{T} \sum_{t=1}^{T} H\left(\phi, \theta_{t}, \theta_{t}^{*}\right) \cdot w_{\phi \mid \gamma_{0}}\left(\theta_{t}, \theta_{t}^{*}\right) \rightarrow \iint \inf _{\phi \in B} H(\phi, x, y) \pi(x) J_{\phi}(x, y) d x d y, \text { a.s. }
$$

holds, for all $\gamma$ in a countable dense set in $\Gamma$. Convergence on compacts is a consequence of epiconvergence and hypoconvergence (see, for example, Geyer, 1994). In order to prove epiconvergence we need to show that

$$
\begin{array}{r}
h(\gamma) \leq \sup _{B \in N(\gamma)} \liminf _{t \rightarrow \infty} \inf _{\phi \in B}\left\{h_{t}\left(\phi \mid \gamma_{0}\right)\right\} \\
h(\gamma) \geq \sup _{B \in N(\gamma)} \limsup _{t \rightarrow \infty} \inf _{\phi \in B}\left\{h_{t}\left(\phi \mid \gamma_{0}\right)\right\},
\end{array}
$$

where $N(\gamma)$ are the neighborhoods of $\gamma$. By topological properties of $\mathbf{R}$, there exist a countable base of open neighborhoods $V_{n}$. By the continuity of $\gamma \rightarrow H(\gamma,) J_{\gamma}$ we can replace the infima by infima over countable sets (e.g., rational numbers). Now construct a sequence $\Gamma_{c}=\left(x_{n}\right)_{n} \in \Gamma$ dense in $\mathbf{R}$ such that, $x_{n}$ satisfies

$$
h\left(x_{n}\right) \leq \inf _{x \in V_{n}} h(x)-\frac{1}{n} .
$$

¿From (13) we have $\lim _{t \rightarrow \infty} h_{t}\left(\gamma \mid \gamma_{0}\right) \rightarrow h(\gamma)$, for all $\gamma \in V_{n} \cap \Gamma_{c}$. Consequently, for all $\gamma \in V_{n} \cap \Gamma_{c}$, and $B \in N(\gamma)$

$$
h(\gamma)=\lim _{t \rightarrow \infty} h_{t}\left(\gamma \mid \gamma_{0}\right) \geq \limsup _{t \rightarrow \infty} \inf _{\phi \in B} h_{t}\left(\phi \mid \gamma_{0}\right)
$$

which implies,

$$
\inf _{\phi \in B \cap \Gamma_{c}}\{h(\phi)\} \geq \limsup _{t \rightarrow \infty} \inf _{\phi \in B} h_{t}(\phi \mid \gamma),
$$

for any $B$ neighborhood of $\gamma$. Take a decreasing collection $B_{n}$ of neighborhoods of $\gamma$ such that $\cap B_{n}=\{\gamma\}$, and we have

$$
\limsup _{n \rightarrow \infty} \inf _{\phi \in B_{n} \cap \Gamma_{c}}\{h(\phi)\} \geq \limsup _{n \rightarrow \infty} \limsup _{t \rightarrow \infty} \inf _{\phi \in B_{n}} h_{t}(\phi \mid \gamma) .
$$

Now (16) reduces to proving that the left-hand side is less then $h(\gamma)$, and follows if $h$ is continuous. The continuity of $H(\gamma,) \cdot J_{\gamma}$, assumption (10) and the dominated convergence theorem

$$
h(\gamma)=\iint\left[\lim _{k \rightarrow \infty} H\left(\gamma_{k}, x, y\right) J_{\gamma_{k}}(x, d y)\right] \pi(x) d x=\lim _{k \rightarrow \infty} \iint\left[H\left(\gamma_{k}, x, y\right) J_{\gamma_{k}}(x, d y)\right] \pi(x) d x=\lim _{k \rightarrow \infty} h\left(\gamma_{k}\right)
$$


yield that $h$ is continuous, concluding the proof of (16).

In order to prove (15) we apply the dominated convergence theorem to get,

$$
\sup _{B_{k}} \mathbf{E}\left[\inf _{\phi \in B_{k}} \frac{H\left(\phi, \theta_{t}, \theta_{t}^{*}\right)}{H\left(\gamma_{0}, \theta_{t}, \theta_{t}^{*}\right)}\right] \rightarrow \mathbf{E}\left[\sup _{B_{k}} \inf _{\phi \in B_{k} \cap \Gamma_{c}} \frac{H\left(\phi, \theta_{t}, \theta_{t}^{*}\right)}{H\left(\gamma_{0}, \theta_{t}, \theta_{t}^{*}\right)}\right]=h(\gamma) .
$$

The hypo-continuity follows from similar arguments.

\section{Proof of Proposition 3}

We need to prove that the assumptions of Proposition 2 are verified. Clearly the continuity assumption is satisfied, and we check now (10). For simplicity, we omit the subscript and use the notation \|\|$=\|\|_{\Sigma^{-1}}$. Fix $\gamma>0$ and $\epsilon>0$ small enough,

$$
\begin{aligned}
& \int \sup _{\phi \in(\gamma-\epsilon, \gamma+\epsilon)}\left(\|y-x\|^{2} \frac{J_{\phi}\left(\|y-x\|^{2}\right)}{J_{\gamma_{0}}\left(\|y-x\|^{2}\right)} \alpha(x, y)\right) J_{\gamma_{0}}\left(\|y-x\|^{2}\right) \pi(x) d y d x \\
& =\iint \sup _{\phi \in(\gamma-\epsilon, \gamma+\epsilon)}\left(J_{\phi}\left(\|y-x\|^{2}\right)\right)\|y-x\|^{2} \alpha(x, y) \pi(x) d y d x \\
& \leq \int\left(\int_{d(\gamma-\epsilon)^{2}<\|y-x\|^{2}<d(\gamma+\epsilon)^{2}} \sup _{\phi \in(\gamma-\epsilon, \gamma+\epsilon)}\left(J_{\phi}(\|y-x\|)\right)\|y-x\|^{2} d y\right) \pi(x) d x \\
& +\int\left(\int_{\|y-x\|^{2} \notin\left(d(\gamma-\epsilon)^{2}, d(\gamma+\epsilon)^{2}\right)} \sup _{\phi \in(\gamma-\epsilon, \gamma+\epsilon)} J_{\phi}(\|y-x\|)\|y-x\|^{2} d y\right) \pi(x) d x .
\end{aligned}
$$

Taking into account that

$$
\sup _{\phi \in(\gamma-\epsilon, \gamma+\epsilon)} \frac{1}{\phi^{d}} \exp \left\{-\frac{\|y-x\|^{2}}{2 \phi^{2}}\right\}=\left\{\begin{array}{cc}
K \frac{1}{\|y-x\|^{2}}, & \|y-x\|^{2} \in\left(d(\gamma-\epsilon)^{2}, d(\gamma+\epsilon)^{2}\right) \\
J_{\gamma-\epsilon}\left(\|y-x\|^{2}\right), & \|y-x\|^{2} \leq d(\gamma+\epsilon)^{2} \\
J_{\gamma+\epsilon}\left(\|y-x\|^{2}\right), & \|y-x\|^{2} \geq d(\gamma-\epsilon)^{2}
\end{array}\right.
$$

with $K>0$, the first integral becomes

$$
\begin{aligned}
& \int\left(\int_{d(\gamma-\epsilon)^{2}<\|y-x\|^{2}<d(\gamma+\epsilon)^{2}} \sup _{\phi \in(\gamma-\epsilon, \gamma+\epsilon)}\left(J_{\phi}(\|y-x\|)\right)\|y-x\|^{2} d y\right) \pi(x) d x \\
& \quad \leq K \int\left(\int_{0<\|y-x\|^{2}<d(\gamma+\epsilon)^{2}} \frac{1}{d(\gamma-\epsilon)^{2}} d y\right) \pi(x) d x \\
& \quad=K \int_{0<\|z\|^{2}<d(\gamma+\epsilon)^{2}} \frac{1}{d(\gamma-\epsilon)^{2}} d z<\infty,
\end{aligned}
$$


and the second integral can be bounded as follows:

$$
\begin{aligned}
\int\left(\int_{\|y-x\|^{2} \notin\left(d(\gamma-\epsilon)^{2}, d(\gamma+\epsilon)^{2}\right)} \sup _{\phi \in(\gamma-\epsilon, \gamma+\epsilon)} J_{\phi}(\|y-x\|)\|y-x\|^{2} d y\right) \pi(x) d x \\
=\int\left(\int_{\|y-x\|^{2} \leq d(\gamma-\epsilon)^{2}} \sup _{\phi \in(\gamma-\epsilon, \gamma+\epsilon)} J_{\phi}(\|y-x\|)\|y-x\|^{2} d y\right) \pi(x) d x \\
+\int\left(\int_{\|y-x\|^{2} \geq d(\gamma+\epsilon)^{2}} \sup _{\phi \in(\gamma-\epsilon, \gamma+\epsilon)} J_{\phi}(\|y-x\|)\|y-x\|^{2} d y\right) \pi(x) d x \\
=\left(\int_{\|y-x\|^{2} \leq d(\gamma-\epsilon)^{2}} J_{\gamma-\epsilon}(\|y-x\|)\|y-x\|^{2} d y\right) \pi(x) d x \\
+\int\left(\int_{\|y-x\|^{2} \geq d(\gamma+\epsilon)^{2}} J_{\gamma+\epsilon}(\|y-x\|)\|y-x\|^{2} d y\right) \pi(x) d x<\infty
\end{aligned}
$$

Combining (17) and (18) proves (10). 

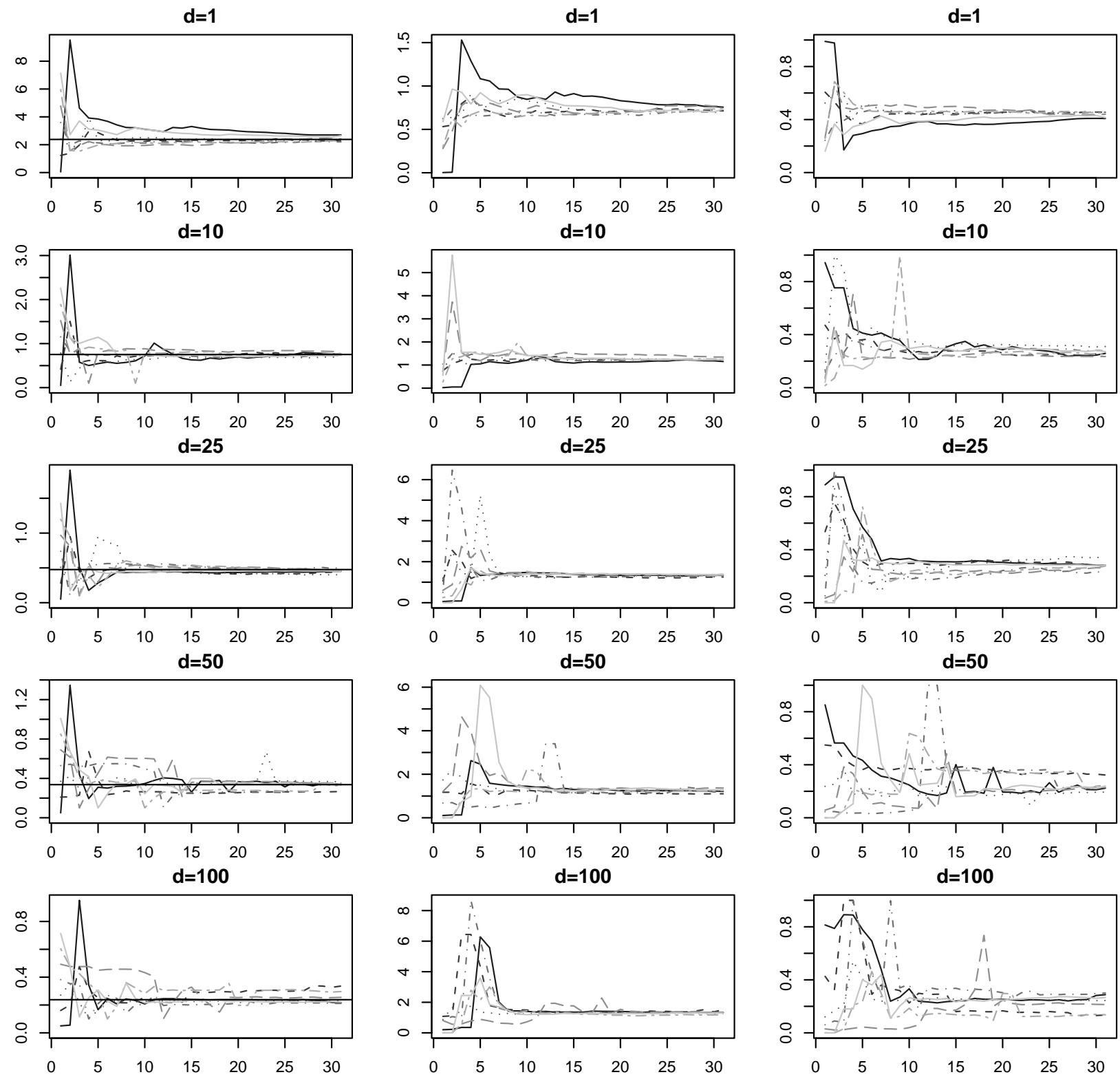

Figure 1: Convergence to the optimal value (solid horizontal line) of the adaptive optimization procedure, given seven equally spaced starting points in the interval $[0,3 * 2.4 / \sqrt{d}], 50$ iterations per step, for dimensions $d=1,10,25,50$, and 100 for the random walk Metropolis algorithm with multivariate normal target distribution. The second and third column of figures show the multiple importance sampling estimator of ESJD and average acceptance probability, respectively. 

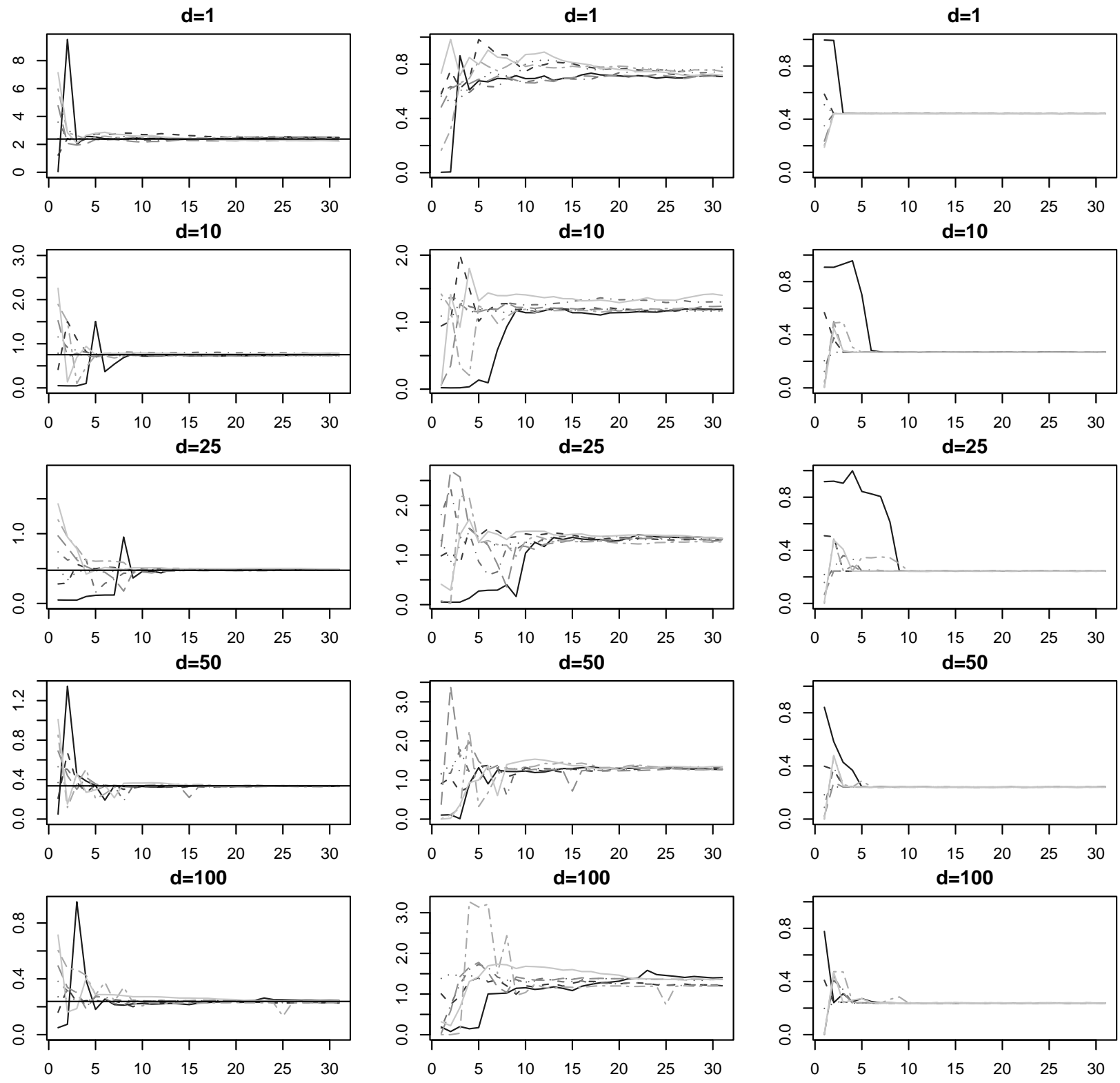

Figure 2: Convergence of the adaptive optimization procedure using as objective the coerced average acceptance probability (to the optimal acceptance value from Figure 1). The second and third column show the multiple importance sampling estimator of the ESJD and average acceptance probability, respectively. Convergence of the optimal scale is faster then optimizing ESJD, although not necessarily to the most efficient jumping kernel (see Figure 5). 
Convergence from high starting value

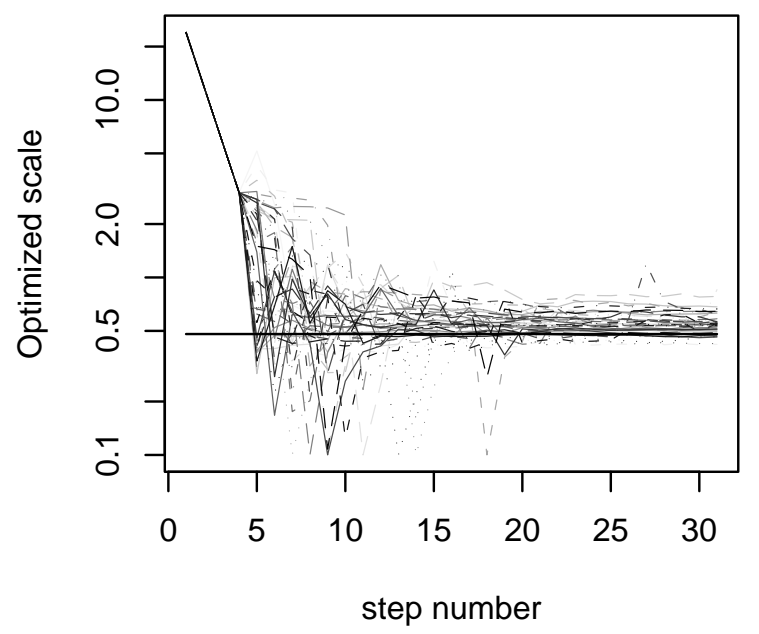

Convergence from low starting value

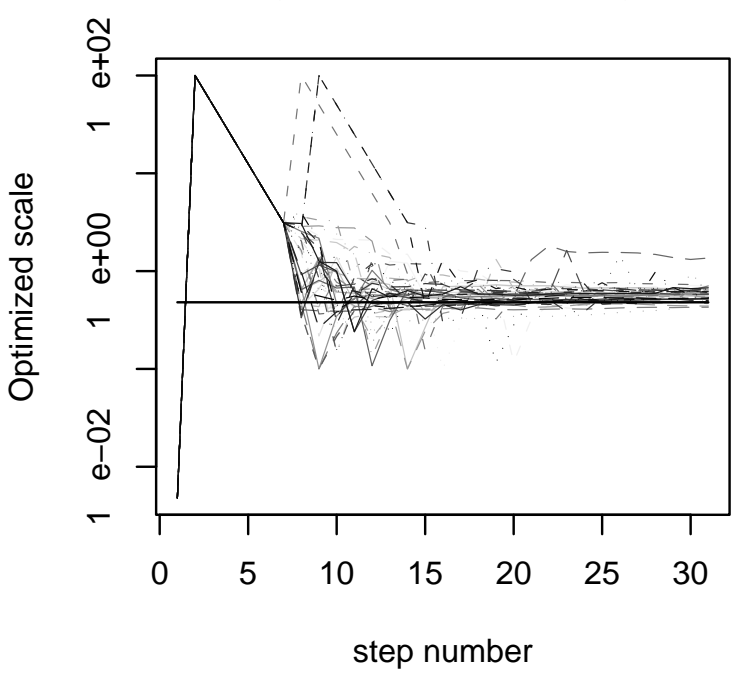

Figure 3: Convergence of the adaptive optimization procedure with extreme starting points of 0.01 and 50 times the optimum, for dimension $d=25$ with multivariate normal target distribution, for 50 independent paths with 50 iterations per steps. The estimated optimal scales are plotted on the logarithmic scale. 
Optimized scale of kernel vs step

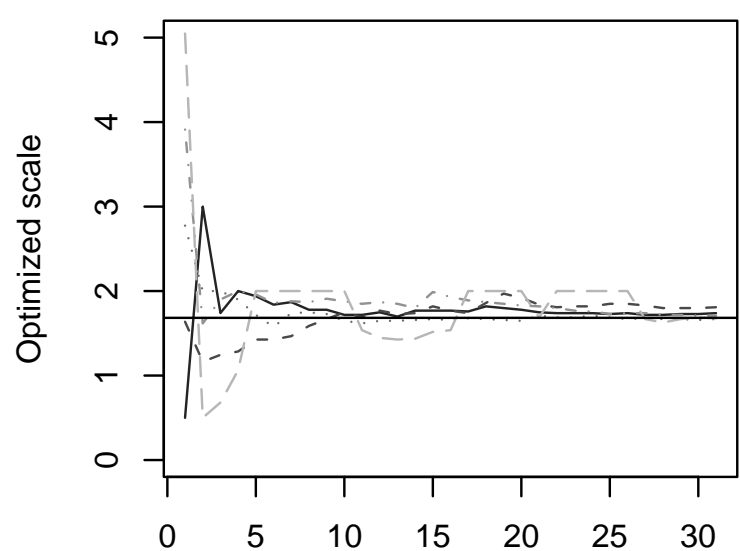

step number

$\operatorname{cov}[1,2]$ vs step

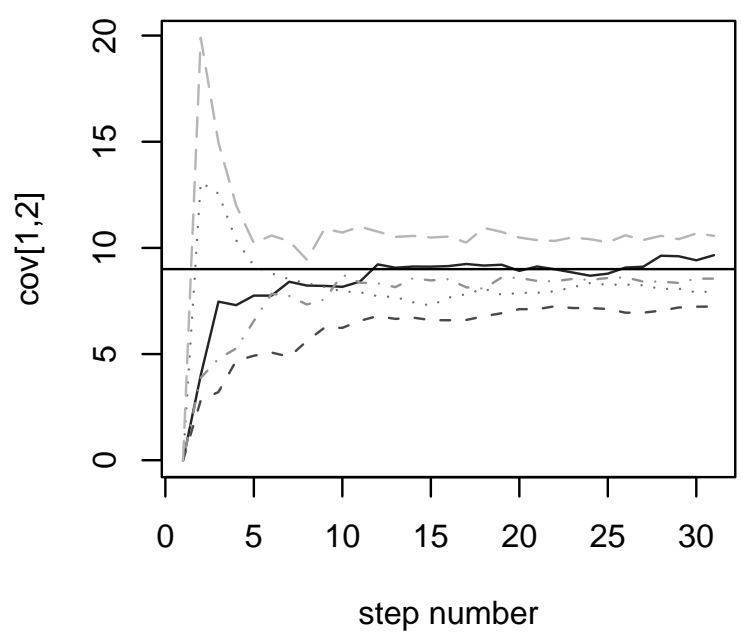

$\operatorname{cov}[1,1]$ vs step

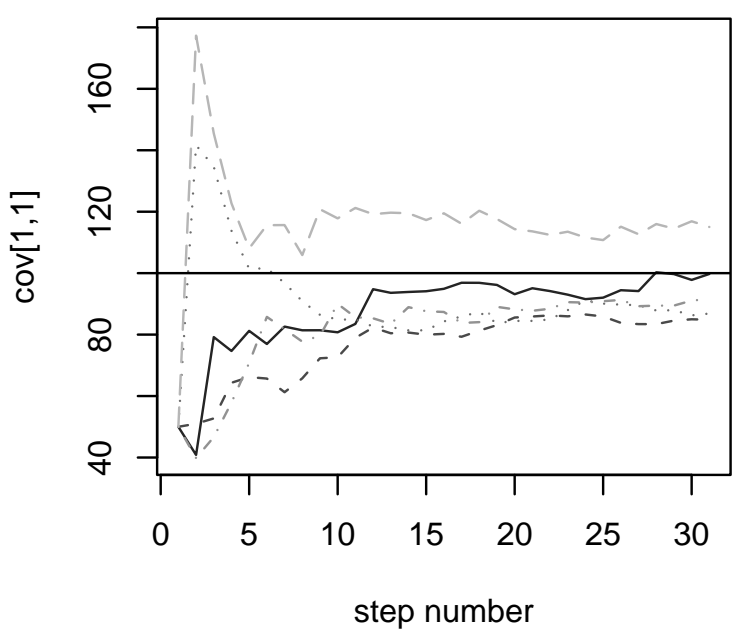

$\operatorname{cov}[2,2]$ vs step

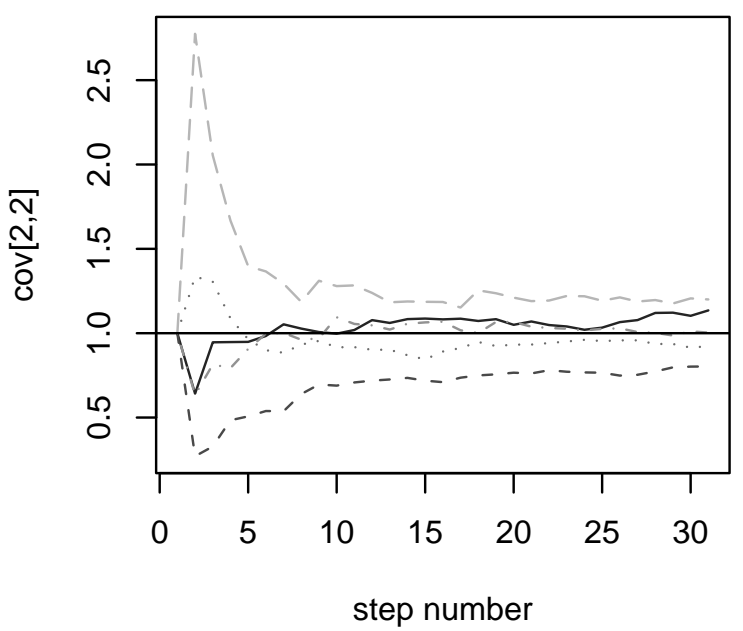

Figure 4: Convergence of the adaptive optimization procedure that maximizes the ESJD by scaling and updating the covariance matrix, starting with independent proposal density with 50 iterations per step. Convergence of the sample covariance matrix is attained in 20 steps and convergence to optimal scaling in 30 steps. 
Maximizing ESJD
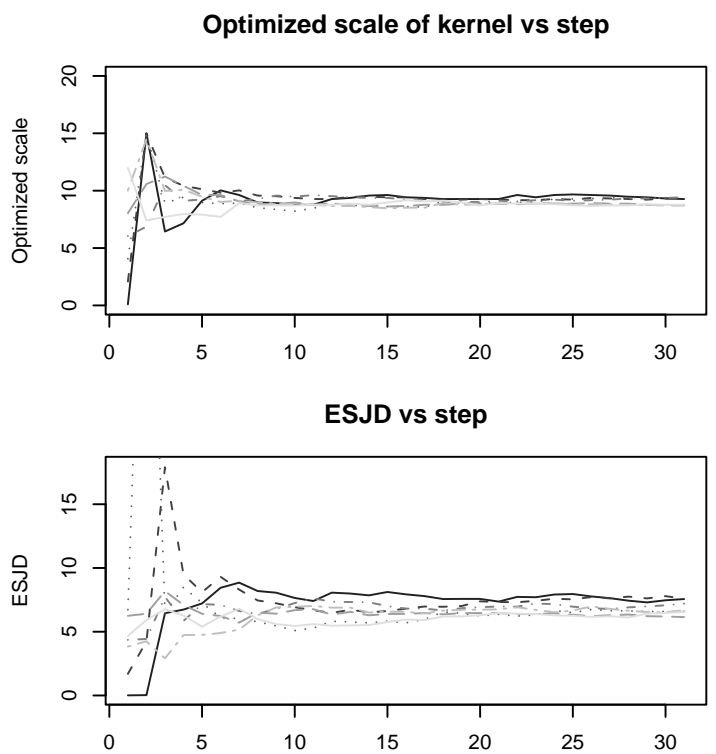

Avg. acceptance prob. vs step
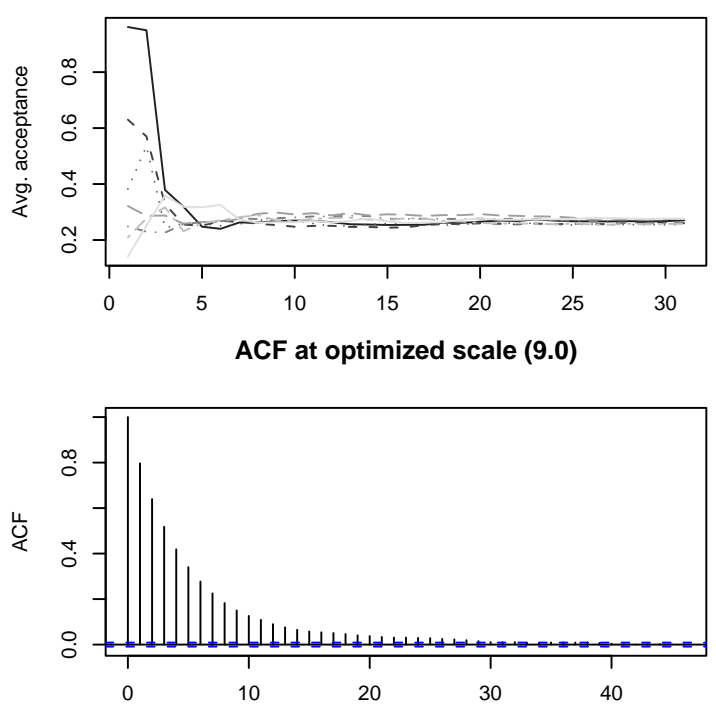

Coercing avg. acceptance prob.

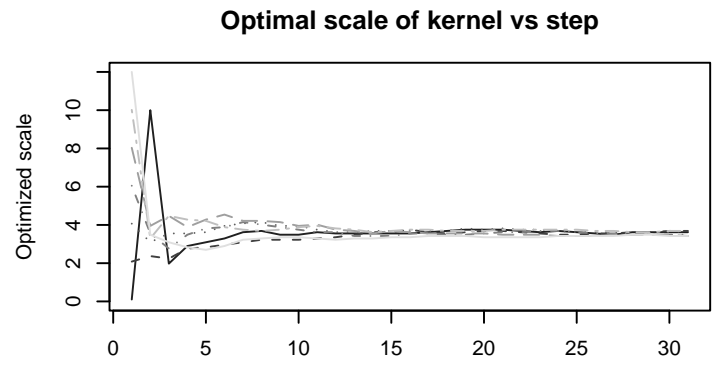

ESJD vs step

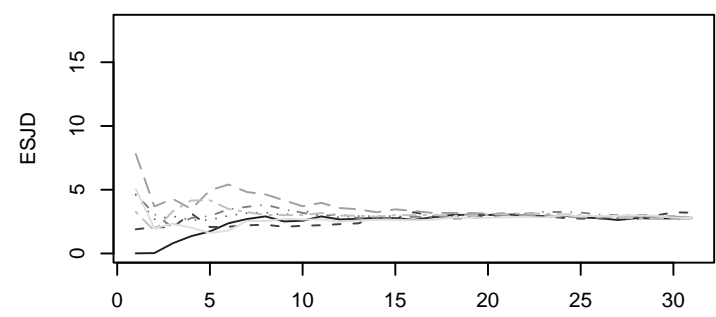

Avg. acceptance prob. vs step
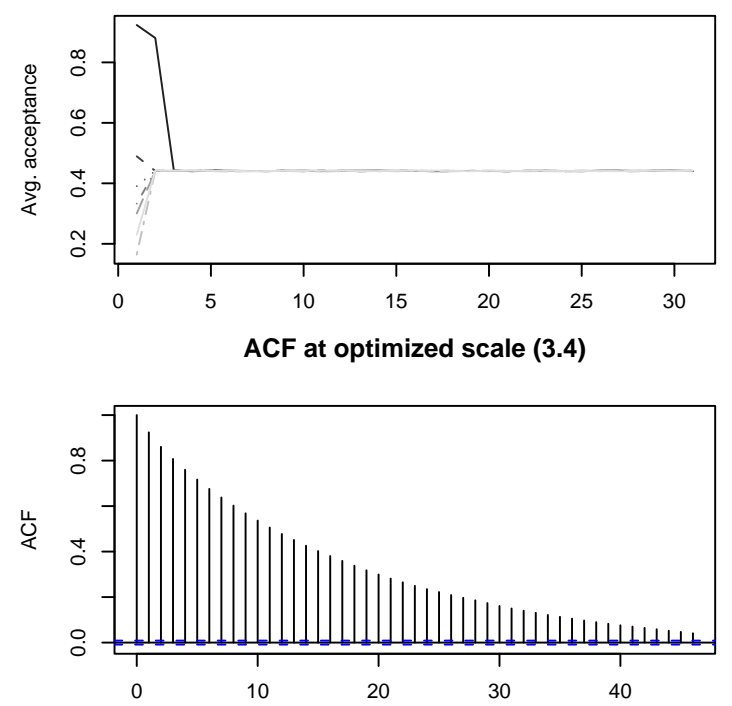

Figure 5: Comparison of two objective functions for the 2-component mixture target of Andrieu and Robert (2001) using our adaptive optimization algorithm: maximizing ESJD (left column of plots), and coercing the acceptance probability to $44 \%$ (right column of plots), with 50 iterations per step. The coerced acceptance probability method converges slightly faster but to a less efficient kernel (see ACF plot). 

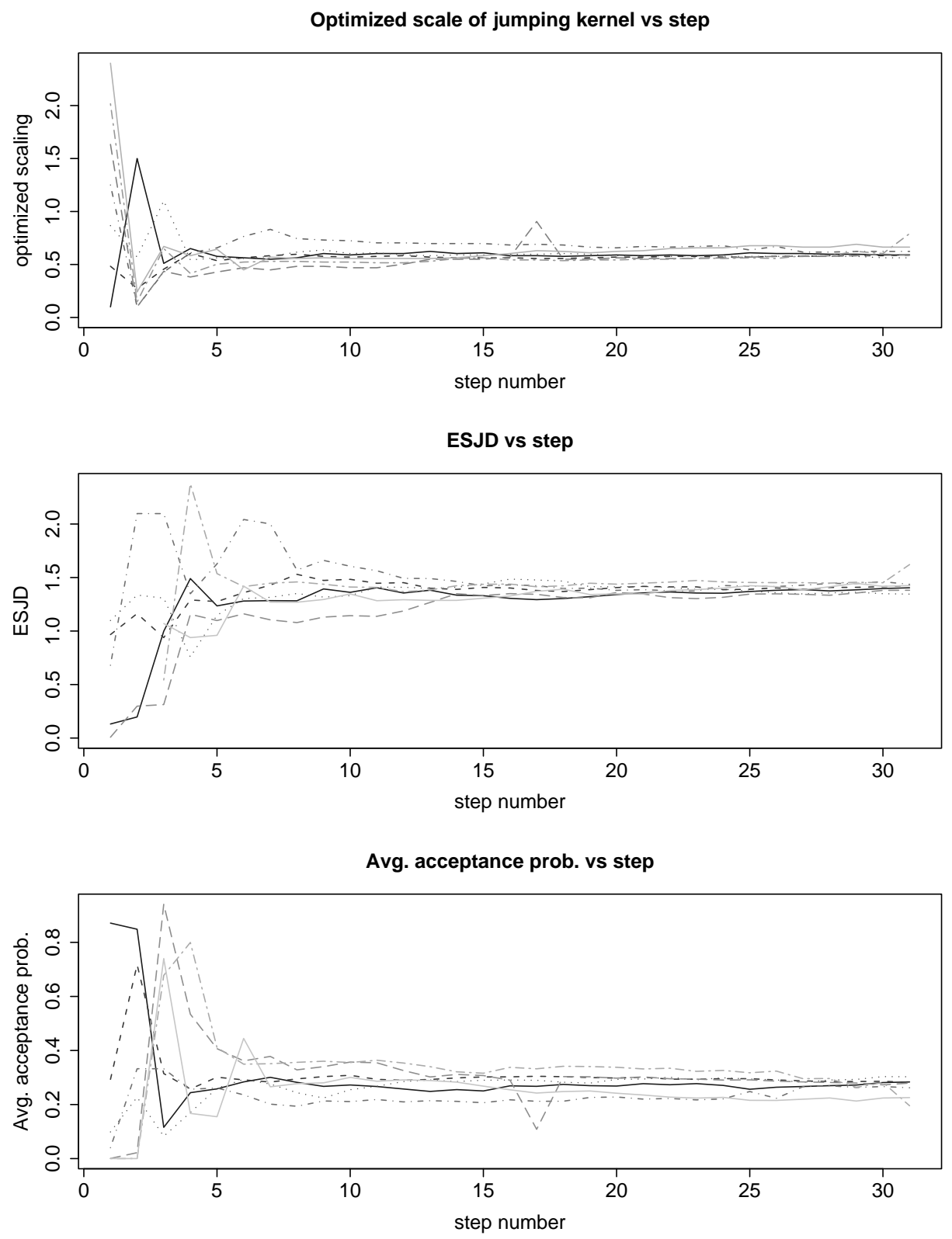

Figure 6: 16-dimensional nonlinear model for a serial dilution experiment from Gelman, Chew, and Shnaidman (2004); convergence to optimal scaling, for seven equally spaced starting values in $[0,2.4]$ with 50 iterations per step and covariance matrix determined by initial optimization. 
Maximizing ESJD
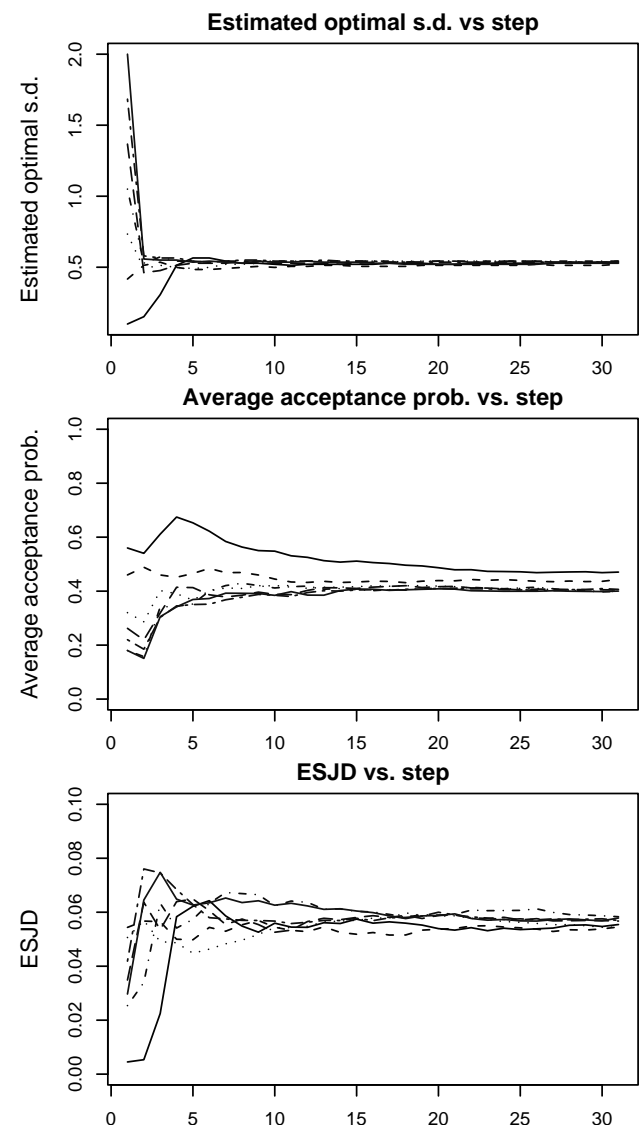

Coerced acceptance probability
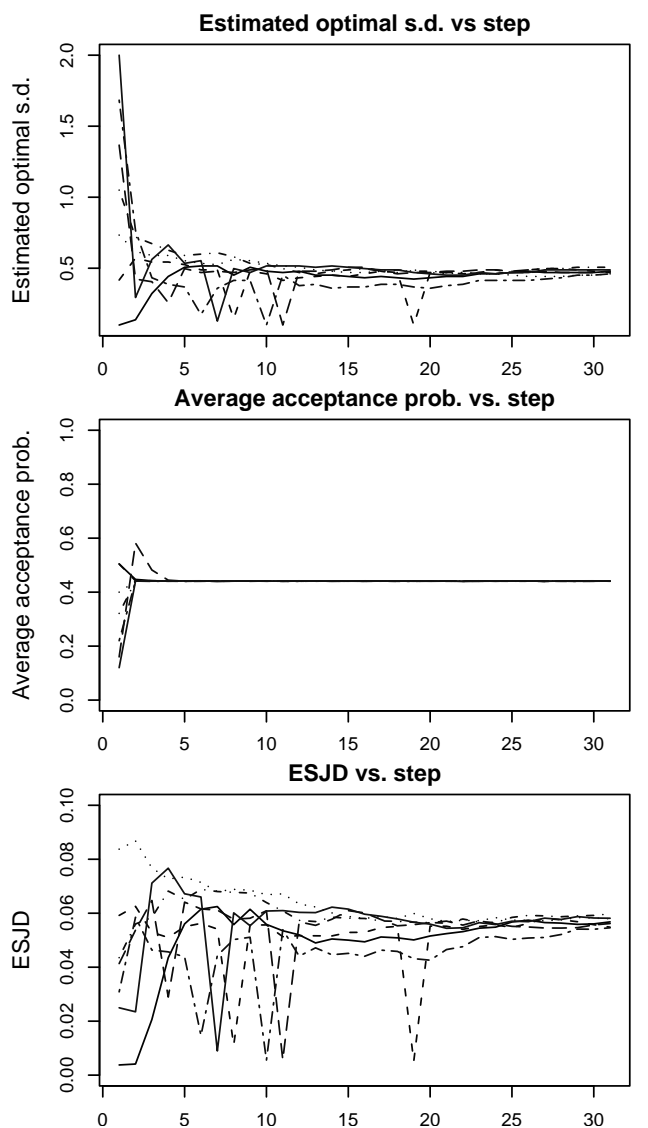

Figure 7: Gibbs sampling with a Metropolis step for the inverse-degrees-of-freedom parameter in the hierarchical $t$ model for the eight schools example of Gelman et al. (2003); convergence of optimal scaling given starting values in $[0,2]$ for two objective functions: maximizing ESJD (left column of plots) and coercing average acceptance probability to $44 \%$ (right column of plots). 